\title{
Gabriela Mistral, der Literaturnobelpreis und der Postmodernismus
}

Wir haben uns im Verlauf des zurückliegenden Teiles unserer Vorlesung auf die unterschiedlichen Aspekte und Ausprägungsformen der literarischen Avantgarden in Europa wie in Hispanoamerika konzentriert und sollten uns nun einigen Überlegungen zuwenden, welche sich aus unserer Arbeit an den Wegen aus der Avantgarde ergeben haben. Bereits der peruanische Theoretiker und Marxist José Carlos Mariátegui, mit dem wir uns im Rahmen unserer Vorlesung aus zeitgründen leider nicht auseinandersetzen konnten, hatte in seinen Siete ensayos de interpretación de la realidad peruana aus dem Jahre 1928 auf eine Reihe zeitgenössischer Entwicklungen rund um eine Gruppe junger Lyrikerinnen aufmerksam gemacht. Letztere lassen sich gewiss nicht unter den Begriff der historischen Avantgarden oder anderer avantgardistischer Strömungen subsumieren. Diese Dichterinnen oder „Poetisas“, so der peruanische Philosoph weiter, hätten einen neuen, frischen Ton insbesondere in die Lyrik Hispanoamerikas eingebracht und die Situation der Literaturen in Peru und weit darüber hinaus stark verändert.

Es war nicht unsere Absicht, durch die Konzentration auf die historischen Avantgarden im spanischsprachigen Amerika in den Hintergrund $\mathrm{zu}$ drängen oder gar zu vergessen, dass es selbstverständlich auch andere Literaten und Literatinnen in der damaligen Literaturszene des Subkontinents gab. Zum Teil sind sie bis heute von großem Interesse und rückten gerade im Zusammenhang einer verstärkten Aufmerksamkeit für die Wege von Schriftstellerinnen als weiblichen „Poetas“ oder „Poetisas“ in den Mittelpunkt öffentlicher Debatten. Im Folgenden möchte ich daher mein Hauptaugenmerk auf jene hispanoamerikanischen Lyrikerinnen richten ${ }^{1}$ und in diesem Zusammenhang drei höchst repräsentative Figuren herausgreifen, welche durchaus unterschiedliche Standpunkte im literarischen Feld der Literaturen Lateinamerikas markieren: Gabriela Mistral, Juana de Ibarbourou und Alfonsina Storni.

Dabei ist das spezifische Interesse, das diesen drei Dichterinnen in den Amerikas wie in Europa entgegengebracht wird, sehr unterschiedlich beschaffen. Die Wege ihrer Rezeption beiderseits des Atlantiks sind nicht leicht miteinander

1 Vgl. allgemein zu den dichterinnen Hispanoamerikas die jüngeren Forschungen von Rodríguez Gutiérrez, Milena: Entre el cacharro doméstico y „la vía láctea“. Poetas cubanas e hispanoamericanas. SEvilla: Iluminaciones Renacimiento 2012; sowie (dies., Hg.): Casa en que nunca he sido extraña. Las poetas hispanoamericanas: identidades, femenismos, poéticas (Siglos XIX - XXI). New York - Bern - Frankfurt am Main - Berlin: Peter Lang 2017. 
vergleichbar. Während die argentinische Lyrikerin Alfonsina Storni vor allem im Kontext feministischer Theoriebildungen in den letzten beiden Jahrzehnten erfreulicherweise erheblich an Bedeutung gewonnen hat, haben die beiden ersteren eher an Terrain verloren. Dies gilt nicht allein für die 1929 zur „Juana de América“ ausgerufenen Juana de Ibarbourou, sondern selbst für die mehrfach preisgekrönte Gabriela Mistral, die übrigens auch einmal den jungen Vicente Huidobro bei einem Dichterwettbewerb in Chile aus dem Rennen warf. Jede dieser drei Figuren steht für eine höchst eigenständige Tradition und darf den Anspruch erheben, zusammen mit weiteren Lyrikerinnen aus der hispanoamerikanischen Welt die ganze Fülle der Dichtkunst jenseits der Avantgarden zu vertreten. Mir scheint es daher wichtig, sie in den Kreis der in unserer Vorlesung behandelten Schriftstellerinnen und Schriftsteller aufzunehmen und dabei gleichzeitig jener Frage nach dem Postmodernismus nachzugehen, welche uns im Kontext unserer Postmoderne-Diskussion interessiert.

Die Tatsache, dass gerade die uruguayische Lyrikerin weitgehend dem Vergessen anheimfiel ist befremdlich, hatte man doch um die Mitte des 20. Jahrhunderts in ihr die große literarische Stimme Hispanoamerikas erblickt. Aber neben Juana de Ibarbourou war im Verlauf der Rezeptionsgeschichte gegen Ende des vorigen Jahrhunderts auch das Interesse an Gabriela Mistral geschwunden. Immerhin war sie es gewesen, die nicht nur die erste Frau, erste Lyrikerin und erste Chilenin sowie Lateinamerikanerin, sondern überhaupt die erste literarische Figur war, die - es war das Epochenjahr 1945 - den Nobelpreis für Literatur erhielt. Auch im deutschen Sprachraum hatte man sich schon früh mit ihr beschäftigt, so etwa unmittelbar nach Ende des Zweiten Weltkriegs der Münchner Professor für romanische Philologie Hans Rheinfelder, der es wie andere Kollegen mehr mit der katholischen Mistral als dem kommunistischen Neruda hielt. Doch die Zukunft gehörte auch in der internationalen Rezeption - zumindest für einen bestimmten Zeitraum - dem Chilenen Pablo Neruda, dessen Stern freilich aus den verschiedensten Gründen längst wieder zu sinken begonnen hat. Noch immer ist es ungewiss, ob seine Landsfrau Gabriela Mistral einmal wieder in der Gunst der Leserinnen und Leser steigen wird, wie sie es wahrlich verdient hätte. Ich möchte Ihnen im Rahmen dieser Vorlesung zumindest eine dichterische Kostprobe aus dem reichen Schaffen der Lyrikerin geben und zugleich eine Lanze für alle drei großen Figuren lateinamerikanischer Dichtkunst brechen.

Zuvor aber einige Hinweise auf das Leben der ersten lateinamerikanischen Nobelpreisträgerin für Literatur. Lucila Godoy Alcayaga hieß die Dichterin eigentlich, die ihren Vornamen zu Ehren Gabriele d'Annunzios und ihren Nachnamen zu denen Frédéric Mistrals, des großen provenzalischen Lyrikers, wählte und unter diesem „nom de plume“ weltweit Berühmtheit erlangte. Sie wurde am 7. April 1889 im chilenischen Vicuña geboren, starb am 10. Januar 1957 in Hemps- 
tead im Staat New York und führte außerhalb der Literatur ein von weiten Reisen und Auslandsaufenthalten erfülltes, höchst aktives und unabhängiges Leben.

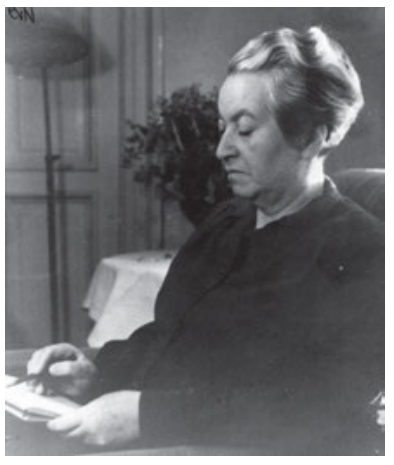

Abb. 87: Gabriela Mistral (Vicuña, Chile, 1889 - Hempstead, New York, 1957).

Schon Gabriela Mistrals Vater war Volksschullehrer gewesen. Er verließ die Familie, als seine Tochter drei Jahre alt war. Sie wurde bereits mit sechzehn Jahren in den regulären Schuldienst aufgenommen und trug so zum finanziellen Überleben der Familie in jenem kleinen andinen Örtchen im Valle de Elqui bei. Ihr beruflicher Aufstieg verlief erstaunlich rasch: Ab 1918 leitete sie nacheinander Schulen und Gymnasien in Punta Arenas, Temuco und Santiago de Chile, wo sie das renommierteste Mädchengymnasium übernahm. Früh hatte sie zu schreiben begonnen. Ihre ersten Texte wurden ab 1905 in Zeitungen der lokalen Presse wie $\mathrm{La} \mathrm{Voz} \mathrm{de} \mathrm{Elqui} \mathrm{veröffentlicht.} \mathrm{Bereits} 1914$ gewann sie für ihre Sonetos de la Muerte den chilenischen Literaturpreis, was sie erstmals in ganz Lateinamerika bekannt machte. Ihre berufliche Unabhängigkeit als Frau und ihre Arbeit als Lyrikerin gingen stets Hand in Hand.

1922 erschien ihr zweiter gefeierter Gedichtband Desolación, der Zeugnis einer großen dichterischen Reife ist und deutlich im Zeichen des Modernismo steht. Ab diesem Jahr lebte sie zumeist außerhalb Chiles, zunächst in Mexiko im Auftrag des mexikanischen Kultusministeriums zwecks der dortigen großen Schulreform unter José Vasconcelos, ab 1925 dann im Auftrag des Völkerbunds, womit ihre Tätigkeiten im diplomatischen Umfeld begannen. Ab 1932 arbeitete sie als chilenische Konsulin in verschiedenen Ländern Europas und Amerikas. Gastdozenturen etwa an der Columbia University in New York schlossen sich an. Sie vertrat ihr Geburtsland Chile in Brasilien, Spanien, Portugal und den Vereinigten Staaten und lebte während des Zweiten Weltkriegs in Brasilien, wo sie mit Stefan Zweig und dessen Frau im Exil bis zu deren Freitod freundschaftlich verbunden war.

Der Nobelpreis für Literatur bildete 1945 sicherlich den Höhepunkt zahlreicher Ehrungen, die der Dichterin national wie vor allem international zuteil- 
wurden. Ihre letzten Lebensjahre standen im Zeichen ihrer Krebserkrankung, an deren Folgen sie in ihrem Haus bei New York verstarb. Doch zuvor hatte sie sich, obwohl sie nun die Öffentlichkeit eher meiden musste, 1954 noch einmal nach Chile begeben, wo sie von ihren Landsleuten begeistert empfangen wurde. Gabriela Mistral wird zwar als chilenische Dichterin gefeiert, die Dimensionen ihres Schreibens sind jedoch ebenso wie ihre Literaturbeziehungen weltweiter Natur, fühlte sie sich doch sehr dem indischen Subkontinent verbunden, dessen

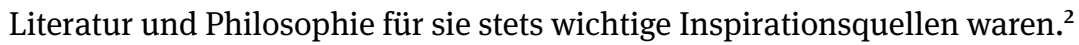

Gabriela Mistrals Dichtkunst beeindruckt durch ihre vermeintliche Schlichtheit, mit der sie sich einen eigenen Platz jenseits der stilisierten Verskunst des Modernismo und der Experimentalpoesie der Avantgarden sicherte. Das Thema der ersehnten Mutterschaft zieht sich durch ihr Werk, eine tiefe Religiosität verbindet sie mit dem Heiligen Franziskus und der spanischen Mystik. Doch ihrer Verbindung mit dem Modernismo blieb sie immer treu, setzte allerdings spezifisch weibliche Akzente, die innerhalb der von Männern beherrschten Bewegung - die Lyrik der bereits früh verstorbenen Kubanerin Juana Borrero dürfte ihr wohl nicht bekannt gewesen sein - eigene Ausdrucksformen schuf.

Dabei bilden die Verzweiflung über den Tod eines Geliebten, die ersehnte Mutterschaft sowie das Thema der Einsamkeit Eckpunkte ihres literarischen Schaffens, in dessen Formen sie - wie vor ihr schon der modernistische Dichter José Martí - auf Elemente der Volksdichtung zurückgriff. Es ist kein Zufall, dass sie dem großen kubanischen Dichter und Revolutionär wunderbare Portraits widmete, in welchen sie ihrer Bewunderung Ausdruck verlieh. Auch sonst bleibt sie den Themenkreisen eines Modernismus Martî’scher Prägung sehr nahe und feiert immer wieder die amerikanische Natur in ihrer Großartigkeit. Selbst die Tendenz zur Askese teilt sie mit dem kubanischen Begründer des Modernismo, dessen Lyrik freilich die minoritäre Traditionslinie modernistischer Verskunst ausmacht. In ihrem letzten großen Gedichtband Lagar von 1954 kulminieren die großen Themen ihrer Lyrik im Schmerz über den Freitod der Zweigs sowie in einer fast mystischen Entrückung, die sich in diesen noch immer mit Desolación zusammenhängenden Gedichten ausspricht. Gabriela Mistrals dichterisches Gesamtwerk steht im Zeichen einer tief empfundenen Menschlichkeit.

In Desolación sind bereits die großen Themen ihrer Lyrik versammelt, wobei ihre Gedichte 1922 noch deutlich im Zeichen des Modernismo stehen. In der

2 Vgl. auch Puppo, María Lucía: Capítulo 18: La danza del perder cuanto tenía. Gabriela Mistral ante Rubén Darío. In: Rodríguez gutiérrez, Milena: Casa en que nunca he sido extraña. Las poetas hispanoamericanas: identidades, femenismos, poéticas (Siglos XIX - XXI). New York - Bern Frankfurt am Main - Berlin: Peter Lang 2017, S. 210-220. 
anschließenden Entwicklung ihrer Dichtkunst wird sich Gabriela Mistral immer weiter vom modernistischen Schreibmodell entfernen. Es mag paradox klingen, doch gelang ihr dies gerade dadurch, dass sie sich mit José Martí an einen der großen Modernisten anlehnte, der in einer häufig kolportierten Begegnung den ihn bewundernden und gut zehn Jahre jüngeren Rubén Darío als seinen ,Sohn“ bezeichnet hatte. Freilich war dieser Sohn in der Folge in die Rolle des prägenden hispanoamerikanischen Modernisten aufgestiegen, der auch nach seinem Tod im Jahre 1916 weiterhin stärksten Einfluss auf die Entwicklung der Dichtung besaß. Demgegenüber stellte die Lyrik Martís so etwas wie eine Unterströmung dichterischen Schaffens im Zeichen des Modernismo dar.

Gabriela Mistral widmete ihm bereits in den zwanziger Jahren einen grundlegenden Essay mit dem Titel Die Sprache José Martís (La lengua de Martí). Martí galt nicht allein die stetige Bewunderung der chilenischen Lyrikerin, sondern auch ein glühender zweiter Vortrag, in dem sie 1938 vor allem auf die Versos sencillos des kubanischen Dichters einging, Verse, die ihr eigenes Schaffen selbst sehr stark prägten. Denn gerade danach war sie stets auf der Suche: Nach einer möglichst geschliffen einfachen Form ihrer Verse, nach einer Nähe zu populären Versformen und Sprechweisen, nach möglichst einfachen Bildern, die nach Martís Vorbild eine komplexe Symbolik zu verbergen vermochten. Vielleicht hat Martí in seiner Lyrik niemals eine bessere Nachfolgerin gefunden als die chilenische Literaturnobelpreisträgerin.

José Martí war als modernistischer Dichter zweifellos das große literarische Vorbild Gabriela Mistrals. Dieses Vorbild zu erreichen und zugleich fortzusetzen gelang ihr auf eine oft verblüffende Weise, und es ist gerade dieser sehr besondere Akzent, auf den ich aufmerksam machen möchte. Oft ist behauptet worden, dass die Lyrik José Martís keine Nachfolger gefunden habe. Ich habe solche Behauptungen auch mit Blick auf die spätere kubanische Dichtkunst schon immer für falsch gehalten. Aber was, wenn diese Nachfolger Nachfolgerinnen gewesen wären? Nicht nur Gabriela Mistral, auch Juana de Ibarbourou hat dem kubanischen Lyriker und Revolutionär aus gutem Grund einen Essay gewidmet. Ihr Werk weist ebenfalls deutliche Züge einer kreativen Anverwandlung Martî’scher Schreib- und Denkformen auf.

Wir können also festhalten: Auf der Suche nach einfachen Formen, nach dem, was José Carlos Mariátegui später als die „Frische“ dieser Lyrik bezeichnen sollte, wurden die hispanoamerikanischen Lyrikerinnen bei José Martí, dem Dichter des Ismaelillo, fündig und spielten damit gleichsam Martí gegen Darío aus, den ,Sohn‘ oder ,hijo‘ des großen Kubaners. Mistral wie Ibarbourou hielten sich fern von jenen berühmten, nach Darío gemodelten modernistischen Schwänen, denen in einem denkwürdigen programmatischen Gedicht bereits 1912 die Gurgel umgedreht worden war. Gewiss war José Martí nur einer ihrer Bezugspunkte, aber eben 
ein besonders wichtiger. Dies macht deutlich, dass die Lyrik dieser großen Dichterinnen nicht aus dem Nichts entstand, sondern durchaus naheliegende Vorläufer besaß. Und zwar solche, die modernistischen Zuschnitts waren und die Position des Postmodernismus nicht als Trennung, sondern eher als Kontinuität und Fortsetzung des Modernismus begreifen ließen.

Dass man diese Kontinuitäten vor allem mit José Martí erkannte, dürfte weniger an der Lyrik des Kubaners als an der Rezeptionsgeschichte dieser Autorinnen liegen, die um die Mitte des 20. Jahrhunderts sicherlich weitaus bekannter waren als César Vallejo, Vicente Huidobro, Virgilio Piñera oder Oliverio Girondo. Doch entwickelte sich die weitere Rezeption dieser „Poetisas“ so, dass sie nach der Jahrhundertmitte - also zum Zeitpunkt der wirklichen Herausbildung einer Literaturwissenschaft außerhalb Lateinamerikas über Lateinamerika - zunehmend in der Versenkung verschwanden und einem weitgehenden Schweigen anheimfielen. Dass hierfür genderspezifische Gründe durchaus eine Rolle spielten, kann nur vermutet werden, ist aber meines Erachtens recht wahrscheinlich. Die Zeiten, in denen sich isoliert ein Hans Rheinfelder mit der Lyrik einer Literaturnobelpreisträgerin nach Ende des Krieges auseinandersetzte, waren vorbei. Auch mussten deutsche Literaturwissenschaftler nicht mehr beweisen, dass sie fest auf dem Boden des Humanismus standen. Wie auch immer wir all diese Entwicklungen bewerten wollen: Die oft geäußerte Behauptung, José Martí habe keine poetischen Nachfolger gefunden, entbehrt gerade mit Blick auf die postmodernistischen Lyrikerinnen jeglicher Grundlage.

Ganz nebenbei sei bemerkt, dass Gabriela Mistral ideologisch keineswegs so einfach von Pablo Neruda zu trennen ist. Denn beide setzten sich 1938 - aus sicherlich unterschiedlichen Horizonten - für die Aufnahme spanischer Bürgerkriegsflüchtlinge ein und überwanden den Widerstand der chilenischen Regierung als wahre und im vollen Wortsinn verstandene Intellektuelle, die sich aus humanitären Gründen für die vom spanischen Franquismus Vertriebenen verantwortungsvoll einsetzten. So kam es zur Aufnahme von wohl zweitausend meist proletarischen spanischen Republikanern, die eine große Anzahl kultureller Aktivitäten in ihrer neuen Heimat Chile entfalten sollten. Doch kehren wir zur Lyrik der Gabriela Mistral zurück!

Die angestrebte Schlichtheit des Ausdrucks erreichte die chilenische Intellektuelle in überzeugendem Maße mit dem Gedichtband Ternura (Zärtlichkeit), der 1924 erschien und eine Sammlung von Kinder- und Wiegenliedern enthielt. Damit war jener Rückgriff auf volkskulturelle Formen sichergestellt, wie ihn Martí bereits in den achtziger Jahren des 19. Jahrhunderts praktiziert hatte. Ein solches Wiegenlied stellt auch das Gedicht Meciendo dar, von dem es übrigens eine bemerkenswert knurrige Selbstaufnahme der chilenischen Dichterin gibt. Dass weder Pablo Neruda noch Gabriela Mistral jene hörbare Meisterschaft eines José 
Lezama Lima bei der akustischen Einspielung eigener Gedichte erreichten, steht an dieser Stelle freilich nicht zur Debatte:

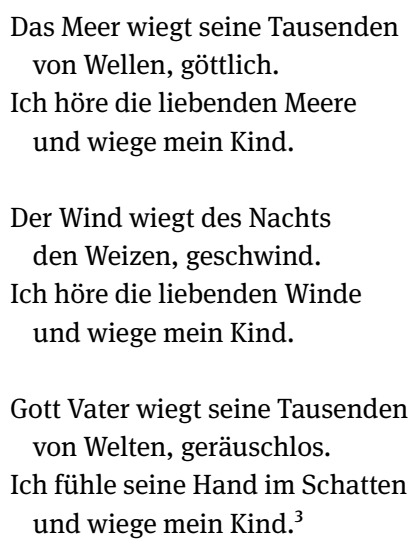

Es sind die einfachen lyrischen Formen, welche in diesem Gedicht im Vordergrund stehen. Es wirkt wie ein spontan von einer wohl weiblichen Gestalt, vielleicht einer Mutter, beim Wiegen ihres Kindes gesungenes Lied, das einen Säugling zum Einschlafen bringen soll. Doch das Gedicht, welches die kinderlose Gabriela Mistral sehr präzise komponiert hat, bringt Wellen, Winde und Welten zusammen mit der Gestalt von Gott Vater, der über alles seine schützende Hand ausbreitet und die Sicherheit von Mutter und Kind garantiert. Schon das Meer ist in der ersten Strophe vom Göttlichen durchdrungen, so wie es auch alle anderen Attribute sind, die mit der Schöpfung verbunden werden: die Wellen, die Winde und die Welten. Ein Gedicht von großer Schlichtheit und ebenso großer Religiosität, das in Gestalt eines Wiegenliedes alle so verschiedenen Welten kosmisch in einer wiegenden Bewegung zusammenführt.

Im Übrigen ist es auch klanglich und nicht nur semantisch um das „mece“, um das Wiegen herum, angeordnet und zeigt eine von Liebe durchdrungene Schöpfung. Diese schließt ebenso die Liebe der Mutter zu ihrem Kind wie die Liebe Gottes zu seiner Schöpfung ein, aber auch alle Elemente einer unermesslichen Natur: die Meeresflächen, die Weizenfelder, die verschiedenen Welten im Universum, die allesamt von der wiegenden Bewegung der Liebe durchzogen sind. Bereits in der ersten Strophe im ersten Vers, in dem sich das „mar“ als Urelement des Wassers und der Fruchtbarkeit klanglich auf die „millares“ der

3 Mistral, Gabriela: Meciendo. In (dies.): Ternura [1924]. Santiago de Chile: Editorial Universitaria 2004, S. 23. 
Wellen hin öffnet, verkörpert sich ein natürliches Wiegen, das sich als göttliches Wiegen erweist, insofern es die letzte Strophe mit der Hand Gottes im „divino“ vorwegnimmt. Diesem göttlich-natürlichen wird das menschliche Wiegen an die Seite gestellt, wobei das Ich mit dem Meer und damit dem Kosmos durch den Klang des Meeres, durch das Hören also, verbunden ist: „oyendo a los mares amantes.“ Dabei werden in den „mares“ zugleich schon fast die „madres“ hörbar, auch wenn wir uns davor hüten sollten, das lyrische Ich ausschließlich weiblich $\mathrm{zu}$ definieren.

In der zweiten Strophe tritt an die Stelle des Elements des Wassers nun das Element der Luft, das bereits den Klang des Meeres transportiert hatte: Den Wasserflächen wird der Luftozean an die Seite gestellt. Die Szenerie wird nun deutlich in der Nacht angesiedelt und zugleich mit einem Element des Lebens, einem Lebensmittel, dem Weizen in Verbindung gebracht: der Wind wiegt die Weizenfelder, welche dem Menschen Nahrung geben. Auch dieses Rauschen und diese Bewegung überlagern und übertragen sich auf das Wiegen des Kindes durch das lyrische Ich und damit auf die Kreatürlichkeit des Menschen. Alles ist in diesen Strophen mit allem harmonisch verbunden.

In der dritten Strophe nun kommt innerhalb dieser so skizzierten Schöpfung Gott Vater hinzu, der seine Tausenden von Welten wiegt, ohne ein Geräusch, ohne einen Klang zu produzieren. Diese Stille, diese Geräuschlosigkeit durchbricht die durch Wiederholung entstandene Ordnung insoweit, als kein Klang auf das lyrische Ich einwirken kann und die ruhige, wiegende Bewegung beziehungsweise den Schlaf des Kindes stört. An die Stelle dieser akustischen Übertragung tritt nun keineswegs eine visuelle, sondern eine taktile Empfindung: das Spüren der Hand Gottes im Schatten, im Verborgenen. Das lyrische Ich braucht Gott Vater nicht zu sehen, um seine Anwesenheit und seinen Schutz zu empfinden.

Dadurch aber wird zugleich die Beziehung zwischen Gott Vater und dem lyrischen Ich zentral, das sich - angespielt seit der ersten Strophe und in Ergänzung von „mar“ und „padre“ klanglich präsent - als Mutter, als weibliches Ich, vorstellen lässt. Denken wir diese Beziehung weiter, so wird das Kind zum Gotteskind, die Mutter gleichsam zu einer neuen Maria, die es im Schutze des Vaters wiegt. Das Kind ist die Frucht einer göttlichen Schöpfung, der sich das Ich gewiss ist und gewiss sein kann: Alles ist im Wiegen der Welt aufgehoben. Das Kind selbst ist damit - wie schon die erste Strophe andeutete, wo „divino“ und „niño“ in derselben Position innerhalb des Verses standen - ein göttliches Kind und damit jener Messias und Heilsbringer, der zwischen Gott und Mensch vermittelt.

Ganz so, wie die Lyrik - und die Lyrikerin - in die Rolle der Vermittlerin zwischen Mensch und Natur, Schöpfung und Schöpfer schlüpft, so sind alle Ebenen der Kreation im Universum wie im Gedicht miteinander tief verbunden. Derartige 
Beziehungen waren schon José Martí keineswegs fremd, suchte er in seiner Lyrik doch immer nach jener schöpferischen Liebe, die alles mit allem zu verbinden vermag. Gleichzeitig stellen wir fest: Welch ein Unterschied zwischen dieser einfachen Sprache in der Dichtkunst Gabriela Mistrals und der stark aufgeladenen, bisweilen überladenen und anspielungsreich-modernistischen Lyrik eines Rubén Darío und mehr noch dessen Epigonen! Die Lyrik des Postmodernismo besetzte in der Tat einen unverkennbaren Platz in der zeitgenössischen Szene der Dichtung. Wir werden sehen, welche Beziehungen sich zwischen diesem Gedicht Gabriela Mistrals und einem der späten Sonette Juana de Ibarbourous herstellen lassen, dem Soneto a Dios, in welchem letztlich das eigene Schaffen innerhalb der Kreation Gottes verankert, damit zugleich relativiert sowie ins Übermenschliche hinaus gesteigert wird.

Es wäre zweifellos möglich, von dieser Ebene aus zu einer selbstreflexiven Metaebene der Lyrik zu gelangen, mit anderen Worten: dieses Gedicht der chilenischen Dichterin als Poetik und Poetologie ihres eigenen dichterischen Tuns zu lesen. Aus dieser Perspektive erschiene dann das göttliche Wiegen als Urmuster jener wiegenden, rhythmischen Bewegung, welche auch das lyrische Ich ausführt und das der Mutter ihre Leben erzeugende und ernährende Kraft gibt. Das Gedicht als Ergebnis dieses Prozesses, dieser göttlichen Verbindung, ist dann die „divina poesía“, die „göttliche Dichtkunst“, die ihrerseits Vermittlerin ist zwischen dem Menschen und dem Übermenschlichen, dem Physischen und dem Metaphysischen, dem Literarischen und dem Metaliterarischen, zwischen Urheber von Geräusch und Bewegung und dem zuhörenden, rezipierenden und wiegenden Ich. Es entsteht ein göttlicher Rhythmus, der die ganze Schöpfung, das göttliche Universum, aber auch das einfache Gedicht durchdringt und verdichtet in einem kleinen Wiegenlied zum Ausdruck bringt.

Ich möchte Ihnen gerne - wie bereits angekündigt - ein weiteres Gedicht von Gabriela Mistral vorstellen. Vorausschicken muss ich nur, dass sich in diesem Gedicht im wörtlichen Sinne alles um eine „Ceiba“ dreht, ein geradezu mythischer und dennoch sehr realer amerikanischer Baum, den Sie vielleicht auch von Abbildungen kennen; ein Baum, den die indigene Bevölkerung der Amerikas stets sehr verehrte und verehrt. Nicht nur in Havanna steht sie am eigentlichen ,Geburtsort' der Stadt und ist innerhalb der kulturellen Symbolik Amerikas bis in unsere Tage von großer Bedeutung und Symbolkraft:

In der Welt, da ist das Licht, und im Licht, da ist die Ceiba, und in der Ceiba ist das grüne Lodern von Amerika! 
Ea, Ceiba, ea, ea!

Ceiba-Baum bist nicht geboren und sie scheint uns allen ewig, Quitos-Indianer pflanzen nicht und die Flüsse wässern nicht.

Sie dreht und wendet sich gen Himmel wie zwanzig wahre Cobraschlangen, und wenn der Wind erst durch sie fährt singt sie wie einstmals Deborah.

Ea, Ceiba, ea, ea!

Es erreicht sie nicht das Vieh und es trifft sie auch kein Pfeil, Angst aber hat vor ihr die Axt, Flammen werden sie nicht verbrennen.

In ihren gebrochenen Ästen krümmt sie plötzlich sich und blutet, danach fällt ihre heilige Milch im Gerinnen und in Strähnen.

Ea, Ceiba, ea, ea!

In dem Schatten der Gigantin tranzen alle jungen Mädchen, und ihre Mütter, die längst tot sind, steigen herab und tanzen mit.

Ea, Ceiba, ea, ea!

Wir reichen unser aller Hände den Lebend'gen und den Toten, und wir drehen uns, drehen uns wir die Frauen, wir die Ceibas ...

In der Welt, da ist das Licht, und im Licht, da ist die Ceiba, in der Ceiba ist das grüne Lodern von der Erde! ${ }^{4}$

4 Mistral, Gabriela: Ronda de la Ceiba ecuatoriana. In (dies.): Ternura, S. $95 \mathrm{f}$. 
Von der ersten Strophe an wird in diesem Gedicht der mythenumrankte Baum der Ceiba mit dem gesamten Kosmos wie mit der Welt Amerikas verbunden. Wie in der biblischen Genesis wird dabei eine Schöpfungsgeschichte mit dem Licht der göttlichen Kreation verknüpft und die Ceiba in den Mittelpunkt dieses Prozesses gestellt. Zugleich ist die Ceiba im tellurischen Element, der Erde Amerikas, verwurzelt und steht stellvertretend für den gesamten amerikanischen Kontinent zwischen Himmel und Erde. Man kommt nicht umhin, hier an Pablo Nerudas Canto General zu denken und an jene Schöpfungsgeschichte der amerikanischen Welt, die auch in dieser „Ronda“ evoziert wird.

Die grüne Ceiba erscheint als Brücke zwischen Himmel und Erde, als Verbindung zwischen den Lebenden und den Toten, verkörpert die mythische Dimension dieser Erde, ist sie doch niemals geboren worden, sondern war immer schon da, ist der Zeit und dem Alterungsprozess enthoben und damit ewig. Die Ceiba ist auch kein Produkt des Menschen, wurde nicht von ihm gepflanzt, und auch kein Produkt der bloßen Natur. Dieser Baum im weiblichen Genus ist im Gegensatz zu anderen Bäumen gleichsam unverwundbar, weder Axt noch Feuer können ihm etwas anhaben. Die Ceiba steht für die weibliche Kraft der Hervorbringung, der Fruchtbarkeit; ihre nahrungsspendenden Eigenschaften kommen in jener Milch zum Ausdruck, welche aus ihrem Inneren fließt. Nicht umsonst wird sie mit den Frauen, die um sie tanzen, assoziiert und erhält dadurch eine generative Dimension, welche in der Beziehung der Ceiba zum Weiblichen, zur Fruchtbarkeit und zum (ewigen) Leben wurzelt.

So ist ihr weibliches Attribut die „santa leche“, die heilige Milch, welche die Milch der Schöpfung und der lebendigen Kraft einer Erneuerung der Welt ist. Daher tanzen die „doncellas“, die Mädchen und Jungfrauen, in einer Ronda um sie herum; selbst ihre verstorbenen Mütter finden sich bei der Ceiba ein und tanzen mit ihren Töchtern, so dass sich Jungfräulichkeit und Mutterschaft im Zeichen ewiger Fruchtbarkeit der Erde miteinander vermengen. Das lyrische Wir in der ersten Person Plural - ist hier zugleich mit den Frauen identifiziert und erkennbar weiblich semantisiert. So wie der Mensch zwischen Himmel und Erde sich aufreckt, nimmt auch die Ceiba jene vertikale Dimension ein, welche Himmel und Erde im Kreis des Lebens umspannt.

Alle maßgeblichen Elemente der Schöpfung sind im Gedicht weiblich, eine Schwierigkeit, die sich jedwedem Übersetzer ins Deutsche - und auch meiner Übertragung - entgegenstellt: „la ceiba“, „la madre“, „la doncella“, „la leche“, „la sombra“, „la mano“, „la luz“, „la tierra“, „la llamarada“, „la América“. Selbst der Wind, „el viento“ singt wie Debora, wobei das biblische Deboralied zu den ältesten Texten der hebräischen Bibel gezählt zu werden pflegt und damit in die (weiblichen) Urzeiten zurückreicht. Debora ist zweifellos eine der wichtigsten Frauengestalten des Alten Testaments und steht mit ihrem mittels propheti- 
scher Gabe ausgeübten Richteramt noch für die matriarchalische und matrilineare Position der Frau ein, die mit der Ceiba verbunden wird. Auch wenn die Welt selbst, „el mundo“, im Spanischen männlich ist, so dominiert in der Ronda de la Ceiba ecuatoriana unübersehbar das weibliche Element die gesamte Schöpfung. Zur Freude des Übersetzers ist „el mundo“ im Deutschen aber weiblich und löst als „die Welt“ alle semantischen Probleme. Die Ceiba ist ohne jeden Zweifel Ausfluss des Göttlichen, der weiblichen Gottheit, und nimmt im matriarchalischen, lebensspendenden Wirken der Milch vegetative Gestalt an.

Es mag sein, dass José Carlos Mariátegui an diese Gedichte Gabriela Mistrals dachte, als er von der neuen starken, frischen Kraft der lyrischen Sprache hispanoamerikanischer Dichterinnen sprach. Sie hätten die zeitgenössische Lyrik umgeformt und zu einer weiblichen Dichtkunst modelliert. Gabriela Mistral hat in ihrem Schaffen ohne Zweifel diese Dimension des Weiblichen und des Mütterlichen wie keine andere Dichterin ausgelotet, stets bestrebt, die Verbindung zwischen Kreatürlichem und Göttlichem über die Kreation, über Geburt und Schöpfung im christlichen Sinne, sichtbar zu machen. Die beiden bislang untersuchten postmodernistischen Gedichte der Chilenin sollten diese Deutung untermauern.

Gabriela Mistrals Verbindung von Göttlichem und Menschlichem (und insbesondere Weiblichem) erscheint bei ihr in aller Schlichtheit, in aller ausdrucksstarken Einfachheit, die auch nach einfachen literarischen Formen verlangt, wie sie vor ihr wohl vor allem José Martí in seinen dem (männlichen) Modernismo verpflichteten Versos sencillos in Hispanoamerika entwickelt hatte. Kein anderer Dichter, keine andere Dichterin hatte sich - soweit ich sehe - nach ihm auf diesen Weg begeben; doch die raffinierter modellierte, aber nicht weniger autobiographisch fundierte Lyrik Rubén Daríos wirkte stärker auf die Nachfahren und Epigonen als die Dichtkunst des Kubaners. Erst mit Gabriela Mistral findet José Martí eine Fortführung der von ihm ausgespannten literarischen Traditionslinien.

Die Chilenin darf gerade in ihrer Verbindung mit der Problematik des Amerikanischen als ideale Vermittlerin der Konzeptionen Martís gelten, auch wenn Pablo Neruda in wiederum ganz anderer Weise ebenfalls dieses poetische Erbe aufnahm und weiterentwickelte. Aus all den bislang aufgeführten Gründen erscheint es mir daher als gerechtfertigt, ja als zwingend, Gabriela Mistral auf Grund ihrer großen Nähe zu einem der führenden Modernisten und einer stets unleugbaren Entfernung zu den historischen Avantgardisten jener Entwicklungsphase des hispanoamerikanischen Modernismo zuzuordnen, die Federico de Onís als Postmodernismo und damit als letzte Phase des literarischen Modernismus bezeichnete. 
Nicht umsonst hatte sich die chilenische Lyrikerin in ihren Essays wiederholt mit dem Kubaner beschäftigt, nicht umsonst hatte sie ihn als „den hombre más puro de nuestra raza“ (als den „reinsten Menschen unserer Kultur“) bezeichnet. Gabriela Mistral ist ohne jeden Zweifel eine Postmodernistin Martí’scher Filiation, durchaus gegenläufig zu Vertretern, die sich (übrigens weitaus in der Mehrzahl) der Tradition ,rubendarianischer Schreib- und Ausdrucksformen zuordnen ließen. Mit Gabriela Mistral findet die hispanoamerikanische Lyrik zu einer Ausdrucksstärke durch Schlichtheit, durch eine semantisch verdichtete „sencillez“, wie sie vor der Literaturnobelpreisträgerin nicht erreicht worden war. Zugleich aber - und darauf sei nachdrücklich hingewiesen - trennt sie ein tiefer Graben von den fast gleichzeitig entstandenen und publizierten Schöpfungen eines César Vallejo oder Vicente Huidobro und selbst noch von ihrem jungen Landsmann Pablo Neruda. Eine größere Nähe ergibt sich hingegen zu Juana de Ibarbourou, die Gabriela Mistral an die Seite zu stellen ist.

Lassen Sie uns zum Abschluss noch einen zugegebenermaßen etwas kurzen Blick auf Gabriela Mistrals Sonetos de la muerte werfen, die sie berühmt machten und von denen ich Ihnen zumindest eines vorstellen möchte! Denn ich will nicht bei Ihnen den Eindruck erwecken oder hinterlassen, dass es sich bei der chilenischen Lyrikerin ,bloß` um eine Dichterin einfacher Bezüge und Beziehungen zwischen Mensch und Gott, Frau und Natur oder zwischen Frau und Mann gehandelt hätte. Dabei ist die Ausgangsstellung ein wenig vergleichbar mit der MutterSohn-Beziehung, welche Gabriela Mistral als reale Autorin nie erlebte oder, in ihrem Sinne, niemals auskosten durfte. Und doch bemerken wir bei ihr eine große existenzielle Distanz, die in ihrer Lyrik zum Ausdruck kommt. Ich möchte Ihnen gleich das erste dieser Todessonette in einer Übersetzung von Albert Theile und Hartmut Köhler vorstellen:

Aus der eiskalten Gruft, in die sie dich gelegt, werde ich dich in die sonnenwarme, schlichte Erde senken. Die Menschen ahnten nicht, dass ich mit dir im gleichen Gelass träumen sollte, auf den gleichen Kissen.

In die sonnenwarme Erde werde ich dich betten, zärtlich wie eine Mutter ihren schlafenden Sohn.

Sanft wird die Erde dich wiegen, wenn sie deinen Leib empfängt, den wunden eines Kindes.

Rosenstaub und Erde werde ich streuen, und in dem linden blauen Dunst des Mondes wird deine leichte Hülle ruhen. 
Besingend meine schöne Rache werd' ich forteilen.

Keines Weibes Hand wird in die Tiefe, die verborgene, reichen, mir dein Gebein, die Handvoll, streitig machen! ${ }^{5}$

Wir haben es in diesem ersten Todessonett der Gabriela Mistral mit - gerade auch im Verhältnis zur Avantgarde der Huidobro und Vallejo, deren hier behandelte Gedichte zum größten Teil in etwa aus dem gleichen Zeitraum stammen - recht traditionell gehaltenen, an kanonische Gedichtstrukturen rückgebundenen lyrischen Ausdrucks- und Bauformen zu tun, wie wir sie aus dem Modernismo kennen. Der Erhabenheit des Sprechens wie auch der Erhabenheit des Sujets entspricht in diesem Soneto de la muerte der spanische Vierzehnsilber, die Form des Alexandriners, die der existenziellen Dimension des Gedichts und dem Schweben zwischen Leben und Tod angemessen ist.

Ebenfalls den Grenzen der lyrischen Tradition ist der hier nur kurz darzustellende Aufbau des Sonetts verpflichtet, insoweit den beiden Quartetten die Exposition, den beiden Terzetten aber die Durchführung überlassen bleibt, wobei die Volte am Schluss in diesem Gedicht ihre semantisch überraschende Ladung erst in den beiden Schlussversen offenbart. Bemerkenswert ist gleichsam auf handwerklicher Ebene, dass mit Blick auf das Reimschema nicht nur die beiden Quartette, sondern auch die beiden Terzette symmetrisch aufeinander bezogen sind und auf diese Weise eine starke innere Beziehung und Zusammengehörigkeit entfalten. Die beiden Quartette greifen auf den Kreuzreim, die „rimas cruzadas“, zurück (abab cdcd), während die beiden Terzette eine andere Reimfolge (ecf ecf) aufweisen und damit über den Reim des ersten Verses des zweiten Quartetts eine Verbindung zu den Terzetten herstellen: „una“ - „cuna“ - „luna“ „ninguna“. Damit fällt umgekehrt der ersten Strophe reimtechnisch eine Sonderrolle zu.

Betrachten wir die bereits im ersten Quartett eingeführten Personen, die „dramatis personae“, dann findet sich eine Spannung einerseits zwischen der zweiten Person Singular und der dritten Person Plural (also zwischen „te“ oder „tú“ einerseits und „los hombres“ andererseits) wie auch ab dem zweiten Vers zwischen der ersten Person und der zweiten Person Singular. Dabei wird schon in der ersten Kombination „te bajaré“ deutlich, dass der ersten Person (dem lyrischen Ich) die aktive, der zweiten Person die passive Rolle zugedacht ist. Deutlich wird dann in einem zweiten Schritt des ersten Quartetts, dass erste und zweite Person Singular

5 Mistral, Gabriela: Los sonetos de la muerte. Die Sonette vom Tode. Übersetzung Albert Theile und Hartmut Köhler. In: Köhler, Hartmut (Hg.): Poesie der Welt: Lateinamerika. Berlin: Propyläen Verlag 1986, S. 130-135. 
klar abgetrennt sind von „los hombres“, das hier keineswegs geschlechtlich definiert wird, sondern allgemein als „die Menschen“ zu übersetzen wäre, was in der hier für Sie ausnahmsweise benutzten deutschen Übersetzung in der Tat der Fall ist.

Diese Abgeschlossenheit von Ich und Du wird von Beginn des zweiten Quartetts an wiederaufgenommen: „Te acostaré“, wobei die verschiedensten Echowirkungen und Lexem-Rekurrenzen in diesem Gedicht schon von Beginn an auffallen: „los hombres - los hombres“, „,soleada - soleada“, „te bajaré - te acostaré“, gleich dreimal „tierra“ u.v.m. Die Beziehung zwischen erster und zweiter Person wird im zweiten Quartett dann recht bald mit der Beziehung zwischen Mutter und Sohn in Verbindung gebracht und damit geschlechtlich wie genetisch determiniert. Zugleich wird das im vierten Vers angelegte gemeinsame Liegen im Bett beziehungsweise auf demselben Kissen zurückgebunden an eine mütterlichkindliche Beziehung, ohne dass dabei die geschlechtlichen und erotischen Konnotationen verschwinden würden: Thanatos ging schon immer mit Eros gemeinsame Wege ...

Das Aufnehmen, die Aufnahme des Körpers des Sohnes, des Kindes durch die Erde („la tierra“, ebenfalls geschlechtlich determiniert), wird hier mit dem „niño dolorido“ in Verbindung gesetzt, so dass die Dimension des Todes nun um jene des körperlichen Schmerzes, vielleicht auch einer lebensgefährlichen Krankheit, erweitert wird. Das lyrische Ich ist weiblich bestimmt und tritt damit in ein weibliches Paradigma ein, zu dem unter anderem „la tierra“, „la madre“, „la cuna“, „la luna“, „la muerte“ sowie weitere Lexeme gehören. Das Wiegen, das wir bereits in Mistrals Wiegenlied kennengelernt hatten, wie auch der Tod sind damit integriert in eine Beziehung, welche in den beiden Quartetten exponiert, in den nachfolgenden Terzetten aber nun entfaltet und in Bewegung gesetzt wird bis hin zum semantischen Höhepunkt, der durch die im Spanischen rahmenden Ausrufezeichen verstärkt wird. Dabei wird der (erotische) Besitzanspruch des Ich auf den Leichnam des Sohnes gegenüber allen anderen Frauen untermauert.

Waren die beiden ersten Strophen von einer Bewegung nach unten, zur Erde hin und in die Erde hinein geprägt, so tritt nun in der dritten Strophe eine Bewegung nach oben hinzu, die himmelwärts zum Mond, zur die Nacht überstrahlenden „luna“ führt. Die tellurische Erdenschwere ist in etwas Leichteres, Lichteres übergegangen. Auch die Erde selbst tritt alsbald in der leichteren Form des Staubes auf, der ebenfalls gleich zweimal erscheint und sogar den Aggregatszustand des Rosenstaubes mitaufnimmt. Die Rosen sind das konventionelle Symbol der Liebe, als vergängliche Blume aber auch Schmerz (die Dornen) und Tod konnotierend. Als Pflanzen bewegen sie sich nach oben hin und stellen damit in gewisser Weise eine Beziehung zwischen unten und oben, Himmel und Erde 
als vertikale Verbindung her. Doch sind sie auch längst schon zu Staub und damit wieder zu einem horizontalen Element geworden und so dem Zyklus von Leben und Tod, Werden und Vergehen zugeordnet.

Die Bewegungen des lyrischen Ich, die in den beiden ersten Strophen aktiv mit jenen des toten Körpers verbunden und in der dritten Strophe in eine nicht zielgerichtete Bewegung übergegangen waren (das Streuen von Rosenstaub und Erde), geht nun in der vierten und letzten Strophe in eine Bewegung über, welche erste und zweite Person Singular voneinander trennt: „Me alejaré.“ Charakteristischerweise ist in diesem Übergang an die Stelle des „te“ das „me“ getreten und zugleich auch ein nach außen getragener Gefühlsausdruck, ein Singen vor dem Hintergrund der eigenen schönen Rache. Doch Rache an wem?

Eben an jenem Ende der Kette von „una“, „cuna“ und „luna“ steht „ninguna“, jene andere Frau, welche an die Stelle von „los hombres“ nun im Singular und in weiblicher Form tritt und folglich den Platz des weiblichen Ich als unverhoffte Rivalin einnehmen könnte. So wird dem „dormirme“ der ersten Strophe letztlich kein „disputarme“ entgegengesetzt: Die Bewegung der Anderen, die ebenfalls in die Tiefe führen würde, wird nicht möglich sein, ist der Weg doch doppelt verstellt: zum einen durch das Ausstreuen von Erde und Staub, zum anderen durch den Weg zur „luna“, zum Mond.

Zugleich ist hier der Weg des toten Körpers, des Leichnams, zu Ende: von der eiskalten Gruft, dem „nicho helado“, in die warme, lebendige Erde, ohne dass die Spuren dieser Umbettung noch sichtbar wären. Tod, Liebe, Geschlechtlichkeit und Leben sind die Spannungspole, an denen sich dieses Gedicht abarbeitet, wobei die erotische Dimension gerade auch auf der Ebene einer Rivalität der Frauen in den beiden letzten Versen eher überraschend herausgearbeitet wird. In diesem Zusammenhang bleibt die Ambiguität zwischen mütterlicher und geschlechtlicher Liebe ebenso bestehen wie der leicht nachvollziehbare Weg von der „cuna“ zur „luna“, Lexeme, die letztlich ebenso eine semantische Polarität eröffnen, wie sie zwischen „una“ und „ninguna“ besteht. Eine ganze Reihe konventioneller Attribute und Symbole überdeterminiert freilich das Element des Todes, das im Schlafen, im Liegen, im Schweigen des Körpers jedoch allgegenwärtig ist.

Mit diesem Todessonett Gabriela Mistrals ist zugleich jene Einheit angesprochen, wie sie im ersten von uns behandelten Gedicht der chilenischen Dichterin zwischen Mensch und Universum, Irdischem und Transzendentem, Mutter und Natur auf erstaunlich schlichte, aber symbolbeladene Weise hergestellt wurde. Der Einklang zwischen Kosmos, Kreatur und Kreation bezieht hierbei ebenso kosmische Elemente wie den Mond, indirekt aber auch die Sonne - das zweifache Auftauchen von „soleada“ ist ein starkes Indiz - mit ein. Auch in dieser wesentlich komplexeren lyrischen Struktur ist der Mensch, ist die Frau eingebunden in den Kosmos ganz so, wie das Gedicht eine polyseme logische Struktur entfaltet, 
in welcher der Mensch im Wiegen zwischen Leben und Tod im doppelten Sinne aufgehoben ist.

Gabriela Mistral wählt in ihren berühmten Sonetos de la muerte eine traditionelle, fast konventionell zu nennende Gedichtform, welche kleine Variationen an der tradierten Form des Sonetts zwar vornimmt, gerade dadurch diese traditionelle Sonettform aber übernimmt und betont. Es gibt in dieser postmodernistischen Lyrik kein avantgardistisches Aufbegehren gegen ein ererbtes Vorbild, gegen das gängige Modell, das sich im Modernismo ganz selbstverständlich der traditionellen Gedichtformen bediente. Dominant ist in dieser Lyrik freilich die Suche nach einfachen Bildern wie auch jene Präsenz der ersten Person Singular, die weiblich determiniert und autobiographisch deutungsleitend angelegt ist. Gegen die ,Selbstverständlichkeit‘ eines männlichen Ich positioniert sich eine weibliche Formensprache, die auf alle Lexeme im Spiegelungsraum des Gedichts übergreift.

Der Unterschied zwischen postmodernistischer Weltsicht und avantgardistischer Formensprache ist eklatant. Die Differenz vor allem zur lyrischen Verarbeitung des Todes beim jungen César Vallejo ist doch sehr beeindruckend: Bei Mistral stellt sich nicht die Frage des Seins, des unergründlichen Schicksals, welche durch keine logische Antwort mehr aufgewogen, ausgefüllt, beantwortet werden kann. Vielmehr ergibt sich eine Dimension allpräsenter Liebe in ihrer zweifachen Form als mütterliche und heterosexuelle Liebe, die das Gedicht als solches überhaupt erst zustande bringt und eine Semantik entfaltet, in welcher der Frau und dem weiblichen Element in Natur und Kultur die zentrale Rolle zukommt. Eben hierin ist im Postmodernismo dann doch eine aufbegehrende Geste auszumachen: eine Revolte gegen die scheinbare Allmacht des Männlichen in der Gesellschaft.

Gabriela Mistral eröffnete 1945 mit ihrem Nobelpreis für Literatur ein halbes Jahrhundert des sichtbaren Aufstiegs lateinamerikanischer Literaturen, der in einer ganzen Serie von Literaturnobelpreisen freilich für ausschließlich männliche Schriftsteller zutage trat und in der zweiten Hälfte des 20. Jahrhunderts mit einer fast schon erdrückenden Dominanz lateinamerikanischer „écriture“ in Zeiten des sogenannten „Boom“ kulminierte. Dies bedeutet nicht, dass die Chilenin gleichsam literarische Mutter eines Gabriel García Márquez, Mario Vargas Llosa oder eines Carlos Fuentes wäre: weit gefehlt! Doch steht die chilenische Dichterin ein für ein gewandeltes Rezeptionsverhältnis in Europa und den USA hinsichtlich der Literaturen des Subkontinents; daher sollte sie in ihrer Funktion als Grande Dame nicht allein der chilenischen, sondern der gesamten lateinamerikanischen Literaturen nicht länger unterschätzt und in ihrer wahren Bedeutung als Meisterin der poetischen Feder verkannt werden. 